\title{
Application of STEM EELS Quantification Relative Compositional Ratio Mapping to Characterize SiCOH - Ultra Low-k Dielectric Materials in Si-based Devices
}

\author{
Wayne W. Zhao ${ }^{1}$ and Michael Gribelyuk ${ }^{2}$ \\ 1. Center for Complex Analyses, GLOBALFOUNDRIES, Malta, New York, USA. \\ 2.Advanced Technology Development, GLOBALFOUNDRIES, Malta, New York, USA.
}

To synchronize with continuous miniaturization of semiconductor device, wafer foundries industry is widely implementing the copper-based interconnects coupled with the ultra-low-k (ULK, $\mathrm{k}=$ dielectric constant) dielectric insulators in the advanced back-end-of-line (BEOL) processes to manufacture Sibased devices. This feature enhances both performance and reliability, such as time-dependent-dielectric breakdown (TDDB) by replacing some $\mathrm{Si}-\mathrm{O}$ bonds with $\mathrm{Si}-\mathrm{C}$ bonds. The $\mathrm{SiOC}(\mathrm{H})$ film is one of typical ULK materials used in BEOL. $\operatorname{SiOC}(\mathrm{H})$ is very sensitive to the electron beam illumination. Therefore development of innovative approaches is crucial for the success to characterize elemental distribution with a high spatial resolution without alteration of the nature of the materials by TEM [1 5]. Effectively handling this technical challenge is becoming one of the stakeholders for the development and implementation of these ULK thin films to facilitate rapid yield learning [5].

Here we demonstrate the effectiveness of STEM-EELS quantification by applying elemental ratio mapping for characterization of small compositional changes in a $\mathrm{SiCO}(\mathrm{H}) \mathrm{ULK}$ film in BEOL. The film was used in SRAM devices, which were built using the 28-nanometer technology node. The electron energy loss spectroscopy (EELS) was used for the 2-D mapping of the elemental composition of $\mathrm{SiCO}(\mathrm{H})$ [6]. To ensure the least damage to the ULK, Gatan Quantum GIF was chosen to minimize the readout time. Elemental ratio maps were generated in the STEM mode using Gatan quantification routines in the Digital Micrograph. The probe corrected FEI Titan was operated at 200KV in the STEM mode with the pixel size in elemental maps $\mathrm{t}=1 \mathrm{~nm}$.

Figure 1a and $1 \mathrm{~b}$ are line profiles extracted from EELS ratio maps of Oxygen versus Carbon for devices at the area of interest (AOI) and at the reference (REF), respectively. Their corresponding HAADFSTEM images are shown in Figures $1 \mathrm{c}$ and 1d, respectively. Figure 1e is a low magnification TEM overview of the devices at AOI and a REF.

It is evident that Oxygen / Carbon ratios were higher in the $\mathrm{SiCOH}$ film at the AOI (ranging from 60 95\%) compared to the Reference site (40 65\%). The composition at the Reference site was deduced by averaging compositions from a number of neighboring sites within the TEM sample. Since the dielectric constant $(\mathrm{k})$ of $\mathrm{Si}-\mathrm{O}$ is higher than that of $\mathrm{Si}-\mathrm{C}$, a higher ratio of $\mathrm{O} / \mathrm{C}$ suggests higher fraction of Si-O bonds in the ULK film at the AOI location. This indicates that the composition of ULK has been altered at the AOI versus the reference. The analysis has helped us explain why the SiCOH film appeared thinner in the defect AOI than in the "good" region at the nearby reference device, and provided insight as a quick feedback to process and integration teams to take corrective actions.

Acknowledgment:

Authors would like to express sincere thanks to Eric Burger for TEM sample preparation. 
References:

[1] W. Zhao, M. Gribelyuk, et al., Microscopy \& Microanalysis, Vol. 21 (Supplement 3), (2015), pp.1036 7.

[2] W. Zhao, et al., Microscopy \& Microanalysis, Vol. 20 (Supplement 3), (2014), pp.362 363.

[3] W. Zhao, et al., Microscopy \& Microanalysis, Vol. 19 (Supplement 2), (2013), pp.902 903.

[4] W. Zhao, M. Gribelyuk, et al., Proc. $38^{\text {th }}$ International Symposium for Testing and Failure Analysis, (2012), pp. 347 355.

[5] W. Zhao, Symp. Proc. the Material Research Society, 2002 Fall Meeting, (2002), Vol. 738, pp. G7.15.1 6.

[6] R. Leapman and J. Hunt, Microscopy, Microanalysis, Microstructure, Vol. 2, (1991) pp. 231-244.
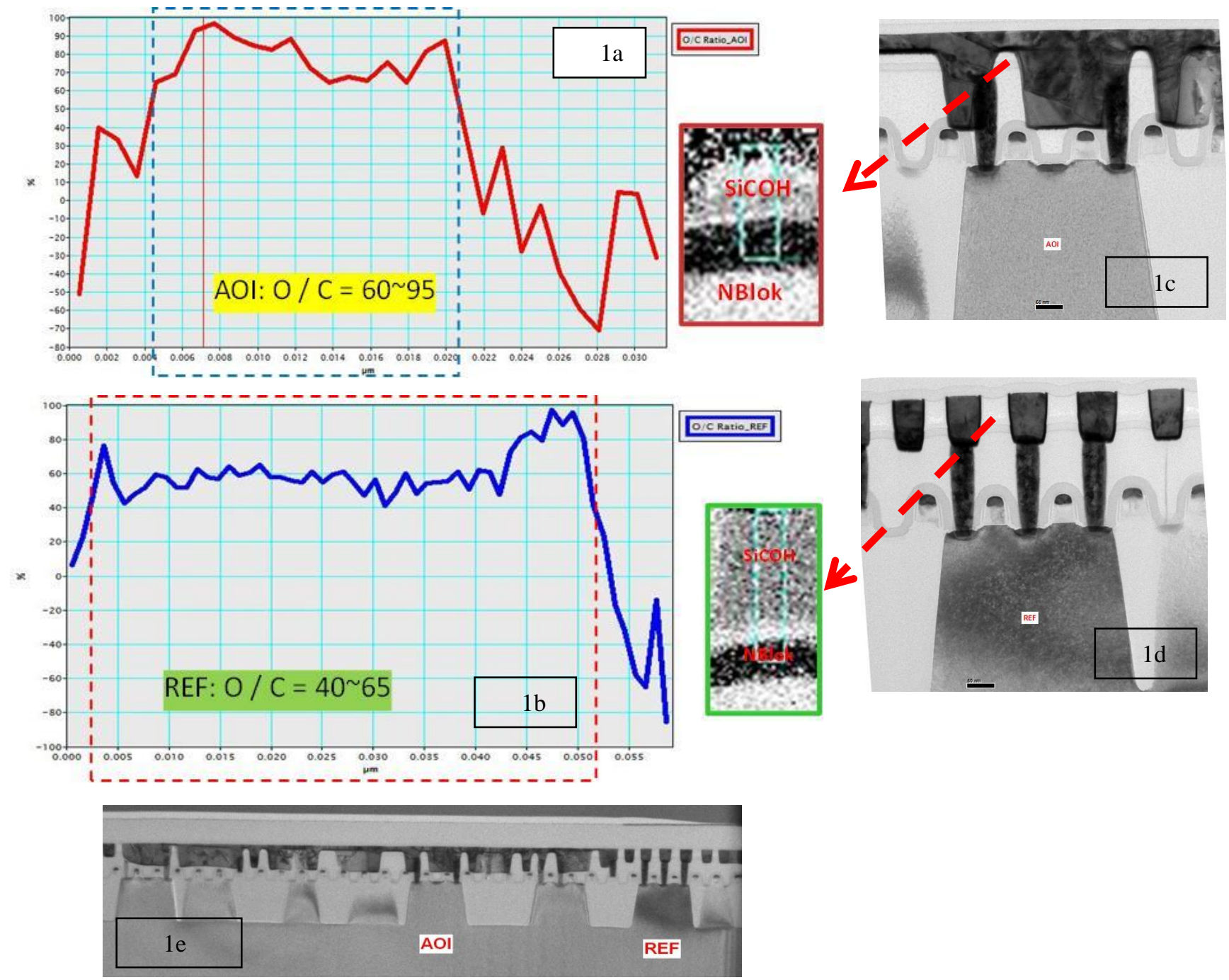

Figure 1. Extracted profiles of Relative Composition Ratio (O / C) from STEM EELS mapping by Digiscan Spectrum Imaging and corresponding HAADF-STEM images, (a) \& (c) at AOI, and (c) \& (d) at REF; (e) a low magnification TEM overview of the devices at AOI and REF. (Note: Left to right in the profiles corresponds to top towards bottom in the EELS ratio mapping.) 